\title{
Responses of Dorsal Cochlear Nucleus Neurons to Signals in the Presence of Modulated Maskers
}

\author{
Veronika Neuert, ${ }^{1}$ Jesko L. Verhey, ${ }^{2}$ and Ian M. Winter ${ }^{1}$ \\ ${ }^{1}$ Centre for the Neural Basis of Hearing, The Physiological Laboratory, Cambridge, CB2 3EG United Kingdom, and ${ }^{2}$ AG Neurosensorik, Carl von Ossietzky \\ Universität Oldenburg, 26111 Oldenburg, Germany
}

\begin{abstract}
The detection of a signal in noise is enhanced when the masking noise is coherently modulated over a wide range of frequencies. This phenomenon, known as comodulation masking release (CMR), has been attributed to across-channel processing; however, the relative contribution of different stages in the auditory system to such across-channel processing is unknown. It has been hypothesized that wideband or lateral inhibition may underlie a physiological correlate of CMR. To further test this hypothesis, we have measured the responses of single units from the dorsal cochlear nucleus in which wideband inhibition is particularly pronounced. Using a sinusoidally amplitude-modulated tone at the best frequency of each unit as a masker, a pure-tone signal was added in the dips of the masker modulation. Flanking bands (FBs, also amplitude-modulated pure tones) were positioned to fall within the inhibitory sidebands of the receptive field of the unit. The FBs were either in phase (comodulated) or out of phase (codeviant) with the on-frequency masker. For the majority of units, the addition of the comodulated FBs produced a strong reduction in the response to the masker modulation, making the signal more salient in the poststimulus time histograms. The change in spike rate in response to the signal between the masker and signal-plus-masker conditions was greatest for the comodulated condition for 29 of 45 units. These results are consistent with the hypothesis that wideband inhibition may play a role in across-channel processing at an early stage in the auditory pathway.
\end{abstract}

Key words: comodulation masking release; auditory scene analysis; response map; auditory brainstem; wideband inhibition; single unit

\section{Introduction}

Many psychophysical masking phenomena can be accounted for by assuming that the auditory system analyzes sound with a bank of overlapping bandpass filters, and that the filter with the largest ratio between signal energy and masker energy determines threshold. However, this idea cannot explain how the detectability of a masked signal can be markedly improved by simultaneously presenting additional, coherently modulated maskers at frequencies remote from the signal frequency (Hall et al., 1984). This phenomenon has been termed "comodulation masking release" (CMR).

Despite extensive study of the CMR paradigm by psychophysicists, little is known of the underlying physiological mechanisms (for review, see Verhey et al., 2003). In the auditory system of the cat, Nelken et al. (1999) suggest that disruption of envelope following is a possible cue for the detection of signals in a modulated background. Nieder and Klump (2001), via recordings in the auditory forebrain of the awake European starling, have also demonstrated a possible physiological correlate of CMR. However, this masking release did not occur when flanking bands (FBs) were positioned beyond the excitatory tuning curves of the units. Although the previous studies were performed at

\footnotetext{
Received Feb. 9, 2004; revised May 7, 2004; accepted May 12, 2004.

This work was supported by the Wellcome Trust.

Correspondence should be addressed to lan M. Winter, Centre for the Neural Basis of Hearing, The Physiological

Laboratory, Downing Street, Cambridge CB2 3EG, UK. E-mail: imw1001@cam.ac.uk.

DOI:10.1523/JNEUROSCI.0450-04.2004

Copyright $\odot 2004$ Society for Neuroscience $\quad$ 0270-6474/04/245789-09\$15.00/0
}

relatively high levels of the auditory pathway, Pressnitzer et al. (2001) have shown that across-channel processing can take place at a level as early as the ventral cochlear nucleus (VCN). They demonstrated that a simple neural circuit involving the wideband inhibition of a narrowband unit could form the basis of a physiological mechanism of CMR. The wideband inhibitor responded well to the amplitude-modulated masker, thus inhibiting the response of the narrowband unit to the same amplitude-modulated masker while leaving the response to the signal relatively unchanged.

A similar circuit, with a wideband inhibitor, has been hypothesized previously to detect spectral notches in complex signals in the dorsal cochlear nucleus (DCN) (Nelken and Young, 1994; Winter and Palmer, 1995; Young and Davis, 2002). Because the inhibition observed in the DCN is stronger than that observed in the VCN, this study tests the wideband inhibition hypothesis of CMR by recording the responses of single units with best frequencies (BFs) of $<5 \mathrm{kHz}$ in the DCN. We found that the response to the signal, as measured by a rate change between the no-signal and signal conditions, was significantly greater for 29 of 45 units when comodulated (CM) FBs were positioned in the inhibitory sidebands. A population analysis of the responses of single units in the DCN showed that type II and type III units have a significantly greater response to the signal in the CM condition compared with the reference (RF) and codeviant (CD) conditions, whereas a group combining type IV and type IV-t units failed to reach significance. The increases in mean discharge rate seen in response to the signal in the comodulated condition sug- 
gest that the DCN may contribute to the physiological mechanisms underlying across-channel processes such as CMR.

\section{Materials and Methods}

Physiology. Experiments were performed on pigmented guinea pigs (Cavia porcellus) weighing between 345 and $550 \mathrm{gm}$. The animals were anesthetized with urethane (1.5 gm/kg, i.p.). Hypnorm (Janssen, High Wycombe, UK) was administered as supplementary analgesia $(1 \mathrm{mg} / \mathrm{kg}$, i.m.). Anesthesia and analgesia were maintained at a depth sufficient to abolish the pedal-withdrawal reflex (front paw). Additional doses of Hypnorm $(1 \mathrm{ml} / \mathrm{kg})$ or urethane $(1 \mathrm{ml})$ were administered on indication. Incisions were preinfiltrated subcutaneously with the local anesthetic Lignocaine (Norbrook Laboratories, Newry, UK). Core temperature was monitored with a rectal probe and maintained at $37^{\circ} \mathrm{C}$ using a thermostatically controlled heating blanket (Harvard Apparatus, Holliston, MA). The trachea was cannulated, and the animal was ventilated artificially with a pump if it showed signs of suppressed respiration. Surgical preparation and recordings took place in a sound-attenuated chamber (Industrial Acoustics, Niederkrüchten, Germany). The animal was placed in a stereotaxic frame that had ear bars coupled to hollow speculas designed for the guinea pig ear. A midsagittal scalp incision was made, and the periosteum and the muscles attached to the temporal and occipital bones were removed. The bone overlaying the left bulla was fenestrated, and a silver-coated wire was placed on the round window of the cochlea to monitor compound action potentials (CAPs). The hole was resealed with petroleum jelly. The CAP threshold was determined at selected frequencies at intervals during the experiments. If the thresholds had deteriorated by $>10 \mathrm{~dB}$ and were nonrecoverable (for example, by removing fluid from the bulla), the experiment was terminated.

A craniotomy was performed, exposing the left cerebellum. The overlying dura was removed, and the exposed brain surface was covered with $1.5 \%$ agar in saline to prevent desiccation. At the end of each experiment, two electric lesions ( $10 \mu \mathrm{A}$ for $10 \mathrm{sec}$ ) were made to enable reconstruction of electrode tracks. The animal received an intraperitoneal overdose of sodium pentobarbitone and was perfused with vascular flush $(0.9 \%$ $\mathrm{NaCl}$ ), followed by fixative ( $1 \%$ glutaraldehyde, $3 \%$ formaldehyde solution). After removal of the brain, the cochlear nucleus was then sectioned and stained with cresyl violet.

The experiments conducted for this study have been performed under the terms and conditions of the project license issued by the United Kingdom Home Office to I.M.W.

Recordings. Single units in the DCN were recorded extracellularly with glass-coated tungsten microelectrodes (Merrill and Ainsworth, 1972). Electrodes were advanced in the sagittal plane by a hydraulic microdrive ( $650 \mathrm{~W}$; David Kopf Instruments, Tujunga, CA) through the cerebellum at an angel of $45^{\circ}$. Single units were isolated using broadband noise and sinusoidal tones as search stimuli. All stimuli were digitally synthesized in real time with a personal computer that was equipped with a DIGI 9636 peripheral component interconnect card connected optically to an analog-to-digital (AD)/digital-to-analog (DA) converter (ADI-8 DS; RME Audio Products, Haimhausen, Germany). The AD-DA converter was used for digital-to-analog conversion of the stimuli as well as for analog-to-digital conversion of the amplified $(\times 1000)$ neural activity. The sample rate was $96 \mathrm{kHz}$. The AD-DA converter was driven using audio streaming input output and a software developer kit from Steinberg (Indianapolis, IN) (Lloyd, 2002).

After digital-to-analog conversion, the stimuli were equalized (phonic graphic equalizer, model EQ 3600; Apple Sound, Flintshire, UK) to compensate for the speaker and coupler frequency response and were fed into a power amplifier (Rotel RB97) and a programmable end attenuator $(0-75 \mathrm{~dB}$ in $5 \mathrm{~dB}$ steps; custom built) before being presented over a speaker [30-1777 tweeter (RadioShack, Fort Worth, TX), assembled by Mike Ravicz, Massachusetts Institute of Technology, Cambridge, MA] that was mounted in the coupler designed for the ear of a guinea pig. The stimuli were acoustically monitored using a condenser microphone (B\&K 4134) attached to a calibrated 1-mm-diameter probe tube that was inserted into the speculum, close to the eardrum. Neural spikes were discriminated in software by a Schmitt trigger using an adjustable thresh- old. Neural activity was stored as spike times on a personal computer and analyzed off-line.

Unit classification. After isolation of a unit, the BF and excitatory threshold of the unit were determined using audiovisual criteria. Spontaneous activity was measured over a $10 \mathrm{sec}$ period. Only units with BFs of $<5 \mathrm{kHz}$ were included in this study.

Single units were classified based on the following: (1) temporal adaptation patterns to suprathreshold BF tone bursts (Young et al., 1988). Peristimulus time histograms (PSTHs) were generated from spike times in response to 250 sweeps of a $50 \mathrm{msec}$ tone at the BF of the unit at 20 and $50 \mathrm{~dB}$ suprathresholds. The rise-fall time of the tones was $1 \mathrm{msec}\left(\cos ^{2}\right.$ gate), and the starting phase was randomized. The tone bursts were repeated for a period of $250 \mathrm{msec}$. (2) Their response map. These maps were generated from the responses to $50 \mathrm{msec}$ tones (repetition period, $200 \mathrm{msec}$ ). The frequency of the tone bursts was varied in 0.1 octave steps from at least 1 octave below $\mathrm{BF}$ to at least 1 octave above $\mathrm{BF}$, and the sound level varied from at least $5 \mathrm{~dB}$ below threshold up to $40-80 \mathrm{~dB}$ above threshold in $5 \mathrm{~dB}$ steps. To reveal inhibitory areas for units that had low spontaneous activity, additional response maps were measured in the presence of a simultaneously gated tone at BF, usually $10 \mathrm{~dB}$ above threshold. (3) Rate-level functions (RLFs) to tones and noise. RLFs were measured from the responses to $50 \mathrm{msec} \mathrm{BF}$ tones or broadband noise (repetition period, $200 \mathrm{msec} ; 10$ repetitions), varying randomly in sound level from below threshold to at least $40 \mathrm{~dB}$ above threshold in $2 \mathrm{~dB}$ steps

Stimuli. The stimuli are the same as used by Pressnitzer et al. (2001) and similar to a paradigm used in several psychophysical studies (Grose and Hall, 1989; Moore et al., 1990; Eddins and Wright, 1994; Hicks and Bacon, 1995; Delahaye, 1999; Meddis et al., 2002). There were three conditions: the RF, CM, and CD conditions, which were all composed of the same signal and on-frequency masker (OFM) but differed in the existence and type of FBs. Figure 1 shows an example of the waveforms (top) and spectra (bottom) of the three stimulus conditions.

The OFM was a pure tone, which was $100 \%$ sinusoidally amplitudemodulated $(\mathrm{SAM})$ at a rate of $10 \mathrm{~Hz}$. Five modulation cycles were presented, resulting in a total duration of $500 \mathrm{msec}$. The carrier frequency of the OFM was normally equal to the BF of the unit, but the OFM was sometimes set to another frequency if the response map showed a much larger range of excitatory levels at this frequency than at BF. OFM level before modulation was at least $25 \mathrm{~dB}$ above the threshold of the unit.

The signal consisted of three $50 \mathrm{msec}$ tone pips, centered in the last three dips of the OFM modulation and with the same frequency as the OFM. The rise-fall time of the tone pips was $25 \mathrm{msec}\left(\cos ^{2}\right.$ gate). The tone pips were always added in phase to the OFM, thus causing an increase in amplitude. The signal level was varied over a range of at least 30 $\mathrm{dB}$ with an additional condition in which no signal was present. All signal-to-masker ratios are expressed as the maximum signal amplitude divided by the maximum OFM amplitude (in decibels). This measure will be referred to as the signal-to-OFM ratio (Verhey et al., 2003).

In the RF condition (Fig. 1, left), only the signal and the OFM were present. In the other two conditions, FBs were added, which were also $100 \%$ SAM tones modulated at a rate of $10 \mathrm{~Hz}$. In the CM condition (Fig. 1 , middle), the FBs were modulated in phase with the OFM, and in the CD condition (Fig. 1, right), the FBs were modulated $180^{\circ}$ out of phase from the OFM. The CD condition gives rise to higher signal detection thresholds in humans than the RF condition, presumably because the codeviant bands exert across-channel masking (Moore et al., 1990; Delahaye, 1999). The level of the FBs was the same as that of the OFM. The number and spacing of the FBs were identical in the CM and the CD conditions and were chosen according to the response map of the unit. If possible, the FBs were placed directly into the inhibitory sidebands of the response area of the unit. The frequency distance between the OFM and the nearest FB was at least twice as large as the spacing between adjacent FBs. If the inhibitory sidebands of the unit were approximately symmetrical, three FBs were placed on either side. However, often the inhibitory sideband on one side of the excitatory area was too narrow to allow the placement of three FBs. If possible, this was compensated for by placing more FBs (four to six) in the other inhibitory sideband. The number of FBs was always between three and six. The frequency spacing between the FBs depended on the width of the inhibitory sidebands of the unit and 

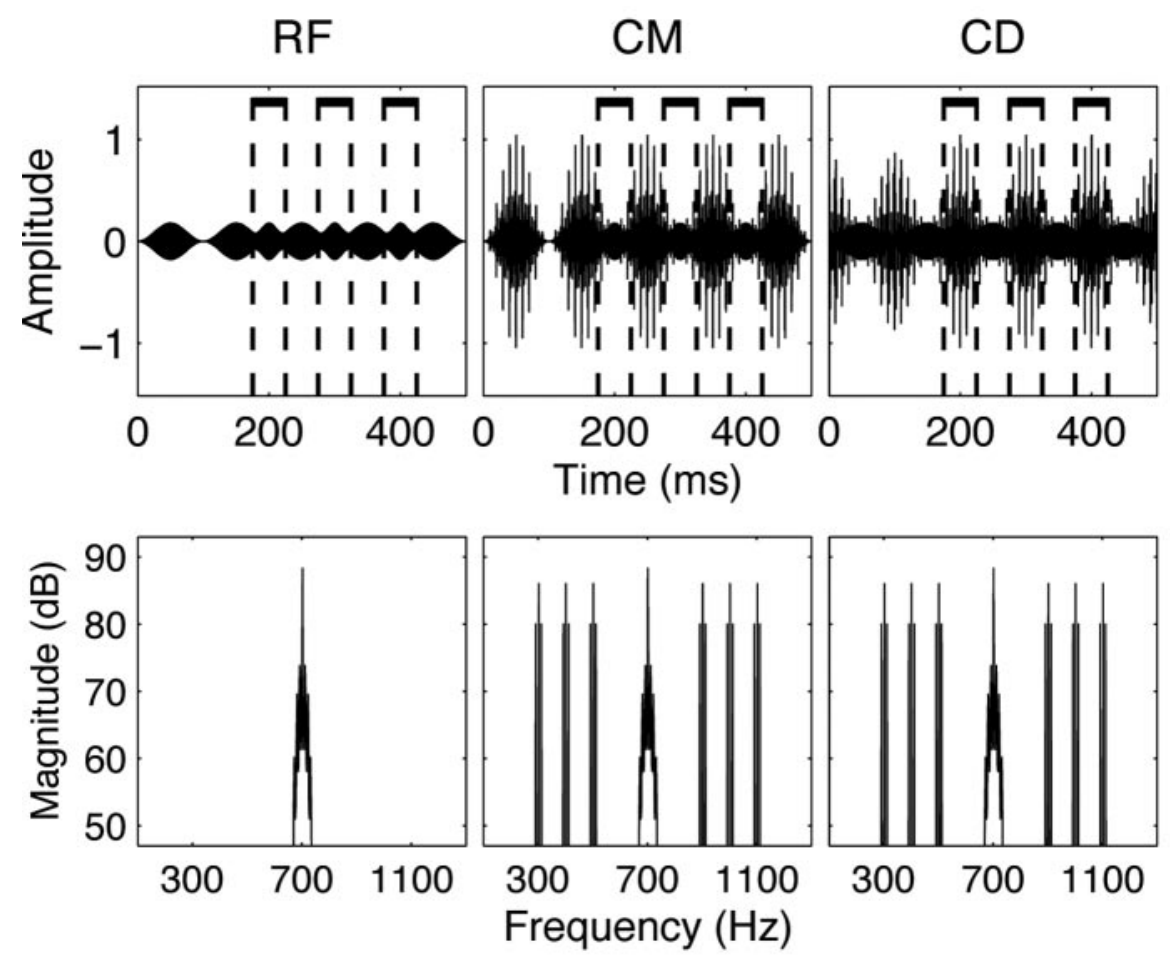

Figure 1. Waveform (top) and spectra (bottom) of the stimuli used in this study. The signal-to-OFM ratio in this example is 0 $\mathrm{dB}$ (defined as the maximum amplitude of the signal pip divided by the amplitude of the carrier of the OFM). Left, RF condition, containing only the signal and the OFM. Middle, CM condition with six FBs added in phase with the OFM envelope. Right, CD condition with six $\mathrm{FBs}$ added $180^{\circ}$ out of phase with the OFM envelope. For each of the top panels, signal position in the waveform is indicated by the bracketed bar between the dotted lines. Signal and OFM frequency in this example are $700 \mathrm{~Hz}$, the spacing of the $F B s$ is $100 \mathrm{~Hz}$, and the frequency separation between the $0 F M$ and the nearest $F B$ is $200 \mathrm{~Hz}$.

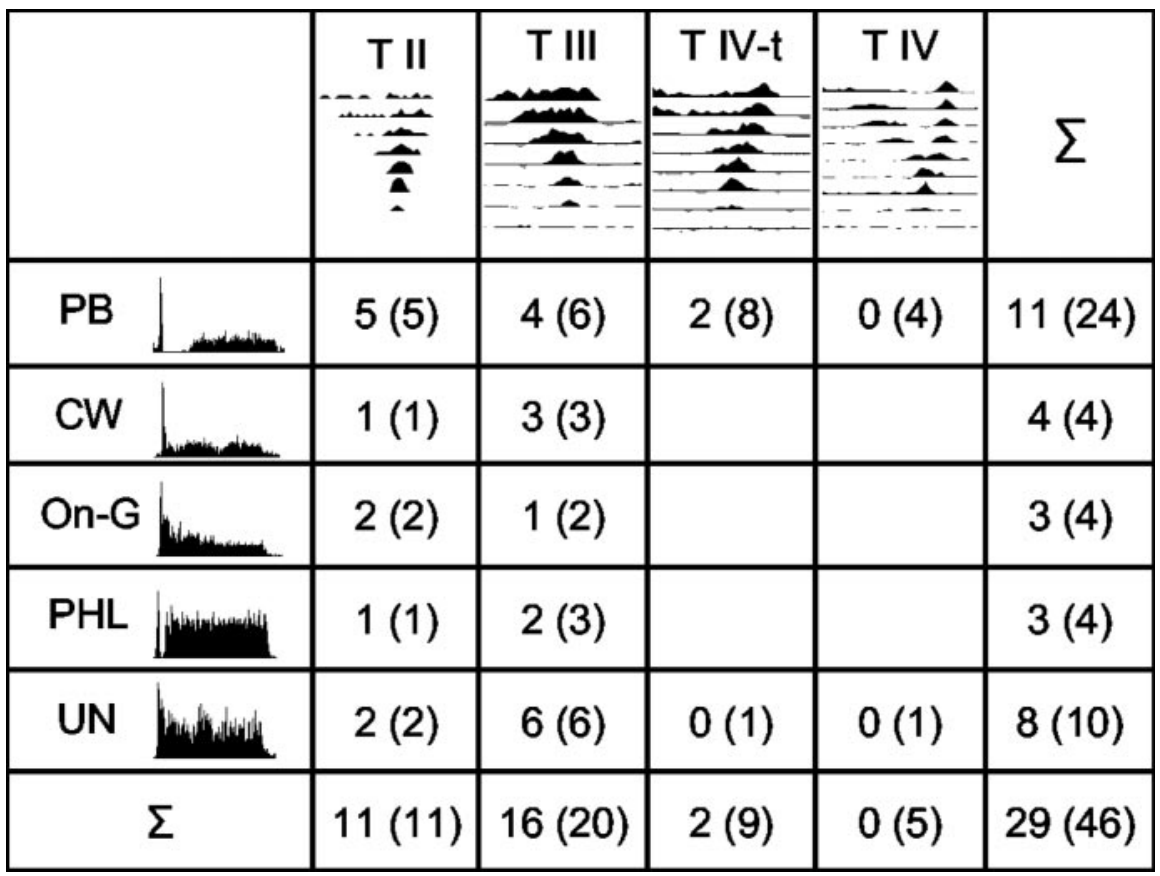

Figure 2. Number of units for which response to the signal was significantly enhanced in the CM condition, relative to the RF and CD conditions, as a function of PSTH (rows) and response map type (columns). The total number of units for each category is given in parentheses, and $\Sigma$ represents the total number of units for each PSTH and response map type. Improvement in signal representation was estimated by the change in mean spike rate in the signal window relative to the no-signal condition (see text). PB, Pause-build; CW, wide chopper; On-G, onset with graded response; PHL, phase-locking; UN, unusual; TII-TIV, response map types II to IV. was chosen to provide the most even spreading of the FBs in the inhibitory sidebands. The most common spacings were 200 and $100 \mathrm{~Hz}$ (44 and $42 \%$ of all experiments, respectively), followed by $300 \mathrm{~Hz}(9 \%)$. A higher $(500 \mathrm{~Hz})$ or lower $(90$ $\mathrm{Hz}$ ) spacing was used for two units.

Each stimulus condition was presented for 50 repetitions with a repetition rate of $1 \mathrm{sec}$. If a unit was lost before 25 repetitions were presented, the data were discarded.

\section{Results}

Recordings were made from 47 single units. The units were assumed to be situated in the DCN for the following reasons: (1) All units were located in the appropriate position along the tonotopic axis of the DCN (i.e., before the abrupt change from low to high best frequencies that indicates the DCN-PVCN border), (2) reconstruction of the electrode tracks showed that all tracks coursed their way through the DCN, and (3) all units had a pause-build (PB) PSTH pattern, a type II or type IV response map, or were located in the same track and immediate vicinity of a pausebuild or type II or type IV unit.

Units were divided into pause-build, chopper, onset, and phase-locking units according to their PSTHs in response to pure tones at BF (Godfrey et al., 1975; Rhode et al., 1983; Shofner and Young, 1985; Rhode and Smith, 1986; Rhode and Kettner, 1987; Rhode, 1999). Examples of the different unit types are shown in the first column of Figure 2. The first row shows a PB. Units were classified as pausebuild if their response consisted of an onset response followed by a decrease and subsequent build-up in firing rate at a 20 or $50 \mathrm{~dB}$ suprathreshold. Units with a build-up response lacking the onset component also fell into the pause-build category. Units that fired regularly (as judged by a coefficient of variation of $<0.5$ ) and showed a characteristic chopping pattern were classified as chopper. The unit sample only contained a wide chopper (first-order interspike interval, $>5 \mathrm{msec}$ ), which is characteristic for the DCN (Fig. 2, second row). An onset response followed by a graded decrease in firing rate denoted an onset with a graded response unit (Fig. 2, third row). If phase locking dominated the response, the unit was classified as phase-locking (Fig. 2, fourth row). Units that did not fall into any of the above-described categories were termed unusual (Fig. 2, bottom row).

In addition to the PSTH classification, units were characterized by their response maps. The response map classification was based on the study by Stabler et al. (1996). The different response map types are shown at the top of Figure 1. If the unit did 
not show any signs of inhibition, it was classified as a type I unit. Recordings were made from one type I unit, but because type I units do not show any inhibition in their response map and the aim of the current study was to investigate the effect of wideband inhibition, this unit was excluded from the present sample. To be classified as a type II unit, the maximum response of the neuron to noise had to be one-third or less of the maximum response of the unit to BF tones. In addition, these units generally had strongly nonmonotonic rate-level functions and no spontaneous activity. Units were classified as type III if they had a V-shaped excitatory response area with inhibitory side bands and gave an excitatory response to noise, the maximum of which exceeded onethird of the maximum response to a $\mathrm{BF}$ tone. For units without spontaneous activity, it is not possible to distinguish inhibitory sidebands from a response map generated using a single tone. These units are therefore commonly classified as a separate category, type I-III. In the current study, a response map in the presence of a low-level excitatory tone burst, usually positioned at $10 \mathrm{~dB}$ above $\mathrm{BF}$ threshold, was routinely measured. This low-level tone burst evoked a small amount of neural ac-

tivity that acted as a surrogate spontaneous discharge rate. This technique allowed determination of the extent and position of inhibitory sidebands. Thus, there was no ambiguity between type I and type III units and there was no need to use the type I-III category. A unit that was driven by a low-level BF tone but was completely inhibited at higher levels (response below spontaneous rate or 0 ) and gave an excitatory response to noise was classified as type IV. These units had large inhibitory areas. Units with response properties intermediate between the type III and type IV response have been classified as type IV-t units. Type IV- $t$ units showed strong signs of inhibition and had nonmonotonic RLFs. The initial peak in the RLF of a type IV-t unit was followed by a decrease in discharge rate, resulting in a reduction of $>25 \%$ at $50 \mathrm{~dB}$ above threshold but not reaching spontaneous rate (Stabler et al., 1996). One unit was classified as unusual on the basis of the response map classification scheme and was also excluded from any additional analyses. Thus, for the remainder of this study, the total number of units was 45 .

The numbers in parentheses in the last column and the bottom row of Figure 2 give the distribution of the different PSTH and response map types, respectively. The majority of units had a pause-build PSTH (23 of 45 units). In the response map classification, type III units occurred most often, followed by type II and type IV-t units.

\section{Responses of single units}

The responses of a DCN unit to the comodulated, reference, and codeviant stimuli are shown in Figure 3, along with the response map and pure-tone PSTH of the unit. This unit was classified as type II in the response map classification scheme. The response map of the unit is shown in the top left corner of the figure. Black areas indicate the excitatory response. Because this unit did not

Type II Unit (1054004)

RF

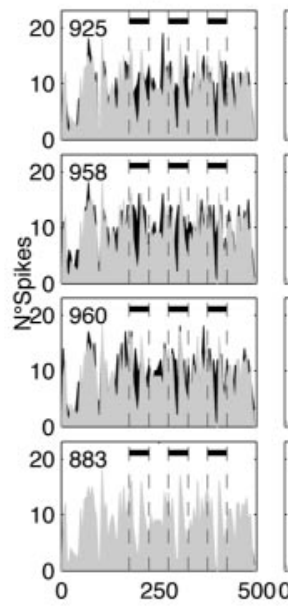

$\mathrm{CM}$

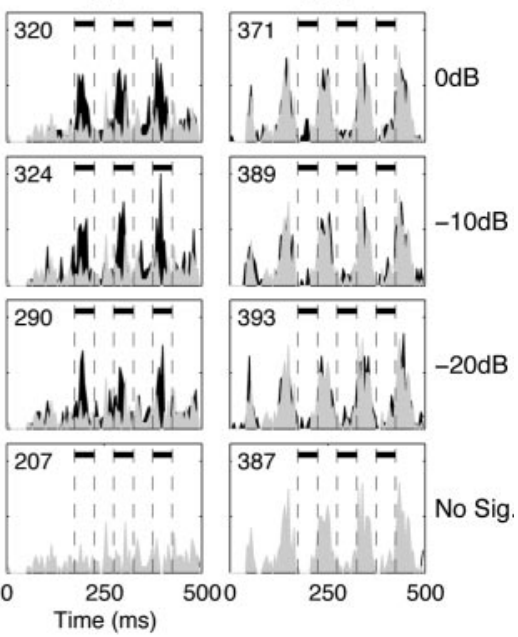

$C D$

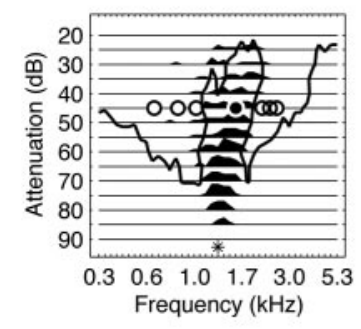

Figure 3. Response map, pure-tone PSTH, and PSTH of the response to 25 repetitions of the three stimulus conditions (right) for a type ll unit (1054004008). The BF of the unit was $1.3 \mathrm{kHz}$, and the spontaneous rate was 0 spikes $/ \mathrm{sec}$. Top left, Response map. The solid line demarcates the boundaries of the sideband inhibition as estimated from a two-tone response map. The filled and BB were set at a level that was $37 \mathrm{~dB}$ above the pure tone threshold. Responses to the RF, $C M$, and $C D$ conditions are shown in the left, middle, and right columns, respectively. The signal-to-OFM ratio increases from bottom to top (as indicated to the right), with the bottom row being the masker-only condition. The temporal position of the signal is indicated by the dashes and filled bars. The total number of spikes is shown in the top left corner of each panel.

have spontaneous activity, the location of inhibitory sidebands was estimated from a two-tone response map and is shown by the solid thick black line. The two-tone response map was measured with an additional low-level excitatory tone, simultaneously gated with the probe tones. A response was regarded as inhibitory if the response to the excitatory tone was suppressed by $>75 \%$. The positions of the signal plus OFM (Fig. 3, filled circle) and the FBs (Fig. 3, open circles) are indicated on the response map. The signal and the OFM were positioned in the excitatory region around the $\mathrm{BF}$, whereas the FBs were primarily positioned in the inhibitory sidebands. Below the response map is the PSTH in response to 250 repetitions of a $50 \mathrm{msec}$ pure tone at $\mathrm{BF}, 50 \mathrm{~dB}$ above threshold. The PSTH of the unit has a build-up shape; therefore, the unit fell into the pause-build category.

The response of the unit to the three stimulus conditions is shown on the right side of the figure. The PSTHs were constructed with a $5 \mathrm{msec}$ bin width. Each column shows the responses as a function of signal-to-OFM ratio for one condition (from left to right: RF, CM, and CD). The BF of the unit was 1.3 $\mathrm{kHz}$, but the signal frequency and OFM were set at $1.6 \mathrm{kHz}$ (Fig. 3 , black circle in response map) because the excitatory response of the unit was greater at this frequency for this particular level. The FBs were set at $0.6,0.8,1,2.2$, and $2.4 \mathrm{kHz}$. The signal position is indicated by the dashed lines in each panel. The signal-toOFM ratio is indicated on the right side of each row, and the total number of spikes is shown in the top left corner of each panel. For this unit, the number of stimulus repeats was 25; for all other examples, the number of repetitions was 50 . The response of the unit to the masker alone (no-signal condition) is shown in the bottom row. In the RF and CD conditions, the modulation of the masker is reflected in the response of the unit. In the CM condition, the response to the masker is greatly reduced relative 
Type III Unit (1046005)
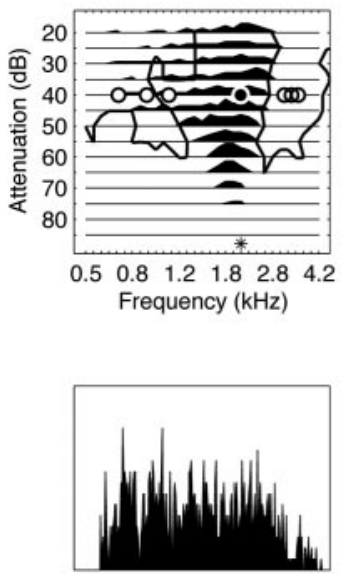

$\mathrm{RF}$
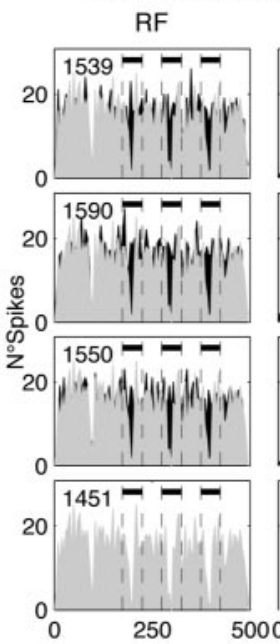

$\mathrm{CM}$
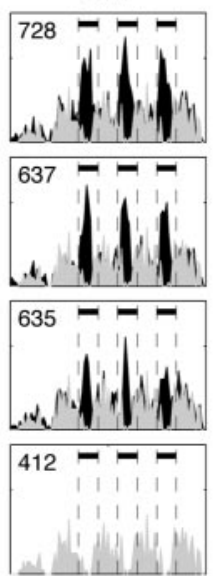

250

Time (ms)

Figure 4. Response map, pure tone PSTH, and PSTHs of the response to 50 repetitions of the three stimulus conditions for a type III unit (1046005012). The layout is the same as in Figure 3. The unit was classified as unusual. Signal and OFM were set at the $\mathrm{BF}(2.1 \mathrm{kHz}$ ) of the unit, the six FBs ( 3 upper, 3 lower) were $200 \mathrm{~Hz}$ apart, and the spacing between the OFM and the nearest FB was $1000 \mathrm{~Hz}$. The OFM and FBs were set at a level that was $36 \mathrm{~dB}$ above the pure tone threshold of the unit.
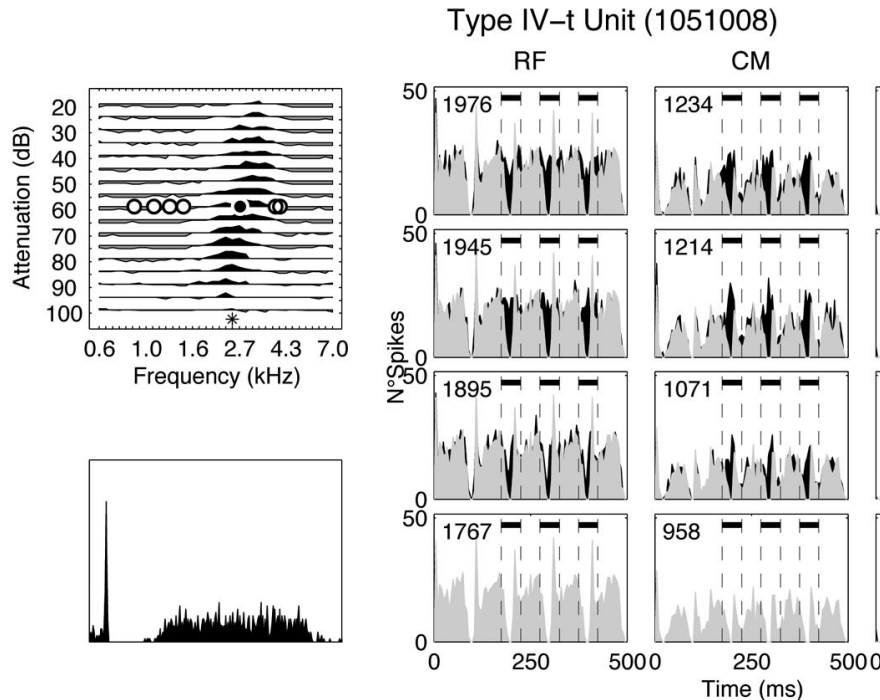

Figure 5. Response map, pure tone PSTH, and PSTHs of the response to 50 repetitions of the three stimulus conditions for a type IV-t unit (1051008011). The layout is the same as in Figure 3. The BF of the unit was $2.5 \mathrm{~Hz}$, and the spontaneous rate was 40 spikes/sec. The unit was classified as pause-build. Signal and OFM were set at $2.7 \mathrm{kHz}$, the six FBs (2 upper, 4 lower) were $200 \mathrm{~Hz}$ apart, and the spacing between the $0 F M$ and the nearest FB was $1200 \mathrm{~Hz}$. The $0 \mathrm{FM}$ and FBs were set at a level that was $33 \mathrm{~dB}$ above the pure tone threshold.

to the RF condition. The response to the masker in the CD condition is also reduced, but the reduction is not as large as in the CM condition (56 and $77 \%$ reduction, respectively, calculated over the entire stimulus duration). In the remaining panels, the response to the masker alone is superimposed in gray over the response to the masker and signal, which is in black. This facilitates comparison between the signal and no-signal conditions, which is the comparison that would have to be made in a psychophysical task. The addition of the signal to the RF condition results in a partial fill-in of the gaps present in the no-signal condition; however, the maximum response to the signal is never stronger than the maximum response to the masker. This is very
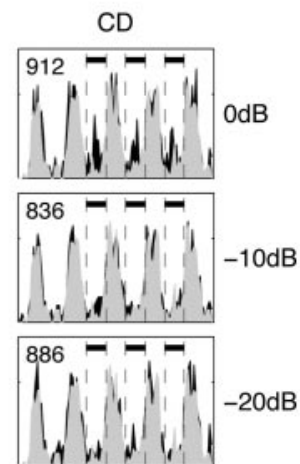

No Sig

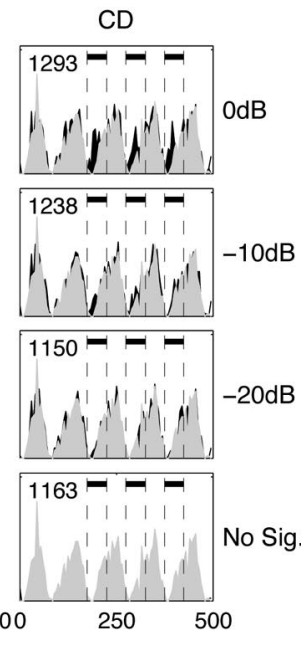

different in the CM condition. Because of the suppression of the response to the masker alone, the signal clearly stands out at all signal-to-OFM ratios. This is also reflected in the increase in the number of spikes with addition of the signal in the different conditions. In the RF condition, the total number of spikes is increased by 9,8 , and $5 \%$ in the $-20,-10$, and $0 \mathrm{~dB}$ conditions, respectively, whereas in the $\mathrm{CM}$ condition, the increase in the total number of spikes was 40,57 , and $55 \%$. There is virtually no increase in spike rate after addition of the signal at any signal-toOFM ratio in the CD condition.

An example of the response of a type III unit to the stimuli is shown in Figure 4. In the PSTH classification scheme, the unit was classified as unusual. The signal frequency was at the BF of the unit $(2.1 \mathrm{kHz})$, and the FBs in the CM and CD conditions were set at $0.7,0.9,1.1,3.1,3.3$, and 3.5 $\mathrm{kHz}$. The response of the type III unit to the three stimulus conditions is similar to the response of the type II unit in many ways. There is a strong reduction (72\%) in the response to the masker alone in the CM condition (Fig. 4, middle column, bottom row) compared with the RF condition (Fig. 4, left column, bottom row), and the response to the signal is much more salient in the CM condition than in the other two conditions. The increase in the number of spikes elicited by the signal ranges between 6 and 10\% in the RF condition, compared with 54,55 , and $77 \%$ in the CM condition $(-20,-10$, and $0 \mathrm{~dB}$ signal-to-OFM ratio, respectively). In the $\mathrm{CD}$ condition, a response to the signal is only present at the highest signal-to-OFM ratios. The increase in total number of spikes was between 4 and 14\%.

Figure 5 shows the responses of a type IV-t unit with a pause-build PSTH pattern. The level of spontaneous rate of this unit allows the inhibitory areas to be seen in the response map without an additional twotone response map. The solid lines indicate the average level of spontaneous rate. Black areas above the solid lines are excitatory responses, whereas gray areas below the solid line show where the spontaneous activity was suppressed. The BF of the unit was $2.5 \mathrm{kHz}$, and the signal and OFM were set at $2.7 \mathrm{kHz}$ (Fig. 5, black circle in response map). The FBs were located at 0.9, 1.1, $1.3,1.5,3.9$, and $4.1 \mathrm{kHz}$; thus, there were four FBs below the signal and OFM and two FBs above the signal, as indicated by the open circles. The response to the masker in the $\mathrm{CM}$ condition is reduced to a lesser extent compared with the other two examples (34\% reduction compared with a 76 and $72 \%$ reduction for the type II and type III unit, respectively). However, the pattern of the results is similar to the other two units, with the response to the signal being more prominent in the CM condition than in the RF condition and nearly absent in the CD condition, apart from the highest level. 


\section{Statistical analyses}

To quantify our findings, a measure based on the increase in spike rate with addition of the signal was used. This method is similar to the one used by Nieder and Klump (2001) in a study on CMR in the starling. The increase in mean spike rate was measured relative to the masker-only condition in three $25 \mathrm{msec}$ windows centered at the center of the signal pips and will be referred to as mean spike change (MSC). The temporal position of the windows was adjusted to take into account the response latency. The mean and SD of the spontaneous discharge rate were calculated in the last $100 \mathrm{msec}$ of each stimulus period. For units with an onset response, the latency was defined as that point at which the activity of the unit exceeded the spontaneous discharge rate plus 2 SDs. For units with a build-up type response, the latency was set at $9 \mathrm{msec}$.

A one-way ANOVA (Matlab 6.5; MathWorks, Natick, MA) was performed to examine the statistical significance of the effects of the stimulus condition at each signal level. For this ANOVA, the spike changes in the $25 \mathrm{msec}$ windows in the responses to the individual sweeps were compared. The dependent variable was the condition (RF, CM, and CD). If the MSC was greatest in the CM condition for at least two consecutive levels and the ANOVA showed a significant effect of stimulus condition, post hoc Scheffé's tests (multcompare; Matlab 6.5) were used to test for significant differences between the individual conditions. If Scheffe's post hoc test showed significant differences in the MSC between the CM and RF and the CM and CD conditions $(p<$ $0.05)$, the signal representation was deemed significantly improved in the CM condition.

According to these criteria, signal representation was significantly improved in the CM condition for 29 of the 45 units (64\%). Many of these units showed enhanced signal representation in the CM condition at all three signal-to-OFM ratios (17 of 29 ). Figure 2 shows the results of the analysis as a function of PSTH type and type of response map. The numbers indicate how many units showed an improved signal representation in the CM condition, and the numbers in parentheses indicate the total number of units in each response map-PSTH combination. All type II and the majority of type III units showed an increased signal representation in the CM condition, whereas the majority of type IV-t and all type IV units failed to show any significant improvement in signal representation with addition of comodulated FBs.

The three examples in Figures 3-5 all showed a significant improvement in signal representation in the CM condition. Figure 6, top, shows the results of the spike rate analysis for these three units. The MSC in the three signal windows after addition of the signal is shown for the signal-to-OFM ratios $-20,-10$, and $0 \mathrm{~dB}$, together with the SE. For all three units, the MSC in the $\mathrm{CM}$ condition is distinctly higher than the MSC in the RF and CD
Type II Type III Type IV-t / IV

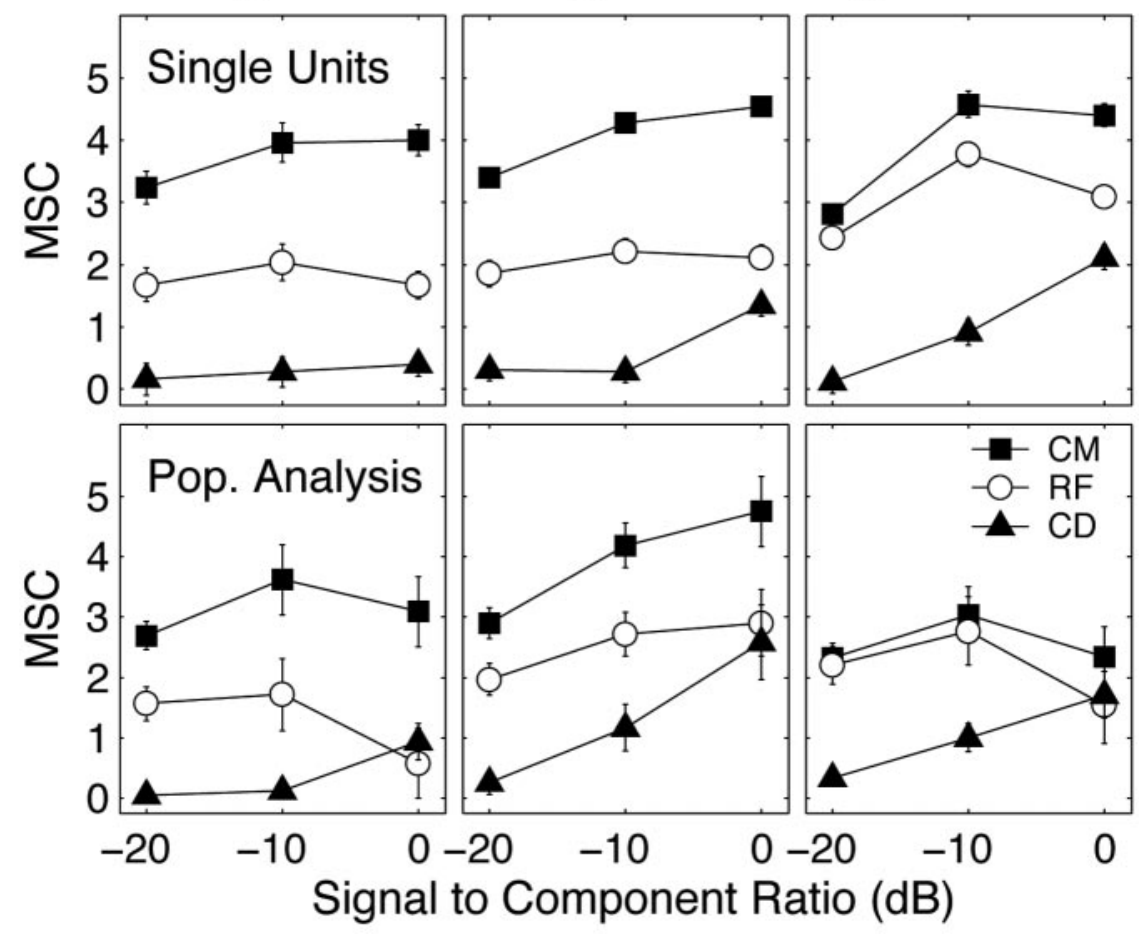

Figure 6. Increase in mean number of spikes (MSC) with addition of the signal. The mean change in spike rate per stimulus mmary of the MSC for type II (left), type III (middle), and type IV-type IV-t units combined (right). The mean and the SE of 11 type II, 20 type III, and 14 type IV-IV-t units are shown. Data are included regardless of whether the responses showed an enhanced response to the signal in the CM condition according to the statistical analysis.

conditions. In the CD condition, the increase in number of spikes is lowest.

\section{Population analyses}

Population analyses were performed on the different response map groups. Because the responses of type IV and IV-t units were quite similar, these two groups were combined into one group, IV-IV-t. Figure 6, bottom, shows the population means and SEs with the left, middle, and right panels showing the average MSCs for type II, III, and IV-IV-t units, respectively. For type II and type III units, the average spike change is clearly highest in the CM condition, but for type IV-IV-t units, the MSCs in the CM and RF conditions overlap. The population average of type II and type III units is quite similar to the results of the single units shown in the left and middle panels of the top row, whereas the performance of the type IV-t unit (right panel, top row) is clearly better than the population average, which is not surprising because the example was one of only two units in that group that showed a significant improvement in signal representation in the CM condition.

For the population analyses, two-way ANOVAs (Anovan, Matlab 6.5; both from MathWorks) were performed with the MSCs of the units in each group and the dependent-variables condition (CM, RF, CD) and signal-to-OFM ratio (-20, - 10, 0). Scheffe's post hoc tests showed that the differences between the MSCs in the CM, RF, or CD groups were significant for type II and type III units $(p<0.00001$ and $p<0.001$, respectively). For the combined group, type IV-IV-t, significance was not reached 
for the comparison of the CM and RF conditions, but there was a significant effect when comparing $\mathrm{CM}$ and $\mathrm{CD}$ conditions $(p<$ $0.001)$. This is reflected in the results for the individual units in each group (Fig. 6, top). Whereas the signal representation was improved for all type II units and 16 of the 20 type III units in the CM condition, this was only the case for 2 of the 14 type IV-IV-t units (both of these units had a type IV-t response map).

Suppression to the masker alone in the CM condition relative to the RF condition was greatest for type II units with a mean reduction in firing rate relative to the RF condition of $61.5 \%$. This mean value was significantly greater than the mean reduction of type III or type IV-IV-t units ( $p<0.001$; two-tailed $t$ test; significant after Bonferroni correction). In contrast, the mean reduction of the latter two groups was virtually identical (32.0 and $32.3 \%$, respectively). However, for units that did not show an enhanced signal representation in the CM condition, the mean reduction of type III units was $14 \%$, whereas the mean reduction of type IV-IV-t units was $32 \%$. This points to a peculiarity of the type IV-IV-t group. In this group, several units did not show a statistically higher increase in firing rate after addition of the signal in the CM condition, despite showing a strong suppression of the response to the CM masker-only condition (33-65\%, 6 units). These units responded only weakly to the signal. Thus, the increase in firing rate was not significant.

Of the 45 units in the sample, only three units did not show a reduced response in the CM masker-only condition. One of these units, classified as type III, nevertheless showed a statistically significant increase in firing rate after addition of the signal in the $\mathrm{CM}$ condition. For this unit, the lower FBs were actually placed in an excitatory region of the response map. This was not true for the other two units (one type III and one type IV-t), although the inhibitory sidebands in which the FBs were placed for these two units were quite weak.

\section{Discussion}

It has been shown that for the majority of type II and type III units in the DCN, the response to an OFM is strongly suppressed if comodulated FBs are added into the inhibitory sidebands of the unit, making the signal more salient than in the absence of FBs or if the FBs were modulated $180^{\circ}$ out of phase (codeviant) with the OFM. Signal representation, estimated by an increase in spike rate, was enhanced for 29 units in the CM condition. The effect observed in this study supports the hypothesis of Pressnitzer et al. (2001) that inhibition plays a role in a physiological correlate of CMR.

\section{Comparison of parameters with psychophysical studies}

In human psychophysics, it has been shown that CMR persists over a range of 2 octaves when the FBs and OFM are equal in level (Cohen, 1991). Most psychophysical studies that have investigated CMR with a FB paradigm have placed the FBs closer to the signal and OFM, with the closest FBs being between 0.2 and 1 octave below and 0.2 and 0.6 octaves above the signal frequency (Schooneveldt and Moore, 1987; Grose and Hall 1989; Schooneveldt and Moore, 1989; Moore et al., 1990; Eddins and Wright, 1994; Hicks and Bacon, 1995). Because the stimuli in our study were adjusted according to the $\mathrm{BF}$ and response map of the unit, the frequency separation between the signal and adjacent FBs varied between experiments. If lower FBs were present, the frequency separation between the OFM and the nearest FB ranged from 0.21 to 2.03 octaves. In $59 \%$ of these experiments, the frequency separation was $\leq 1$ octave. The frequency separation between the OFM and the upper FBs was between 0.35 and 2 oc-

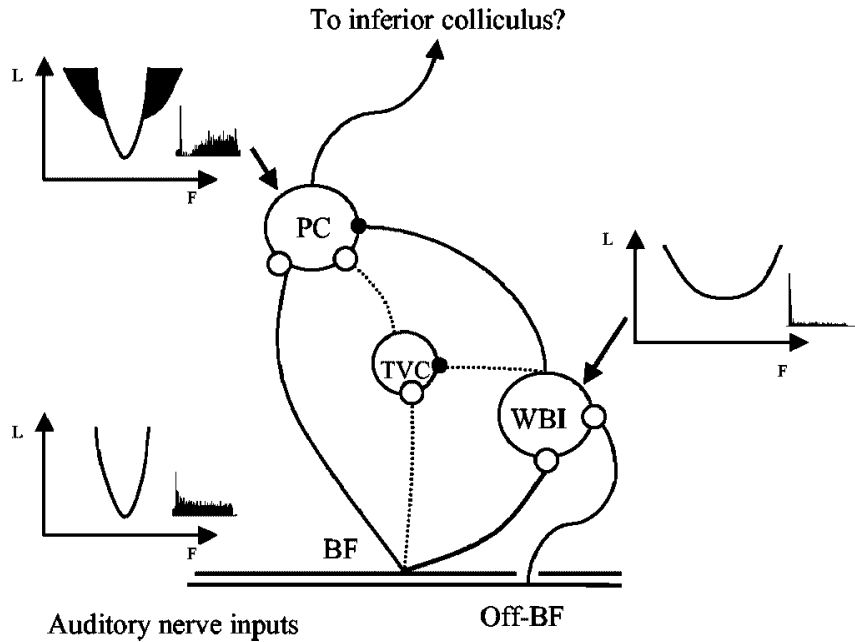

Figure 7. Possible neural circuit to explain CMR, originally proposed by Pressnitzer et al. (2001), modified by Meddis et al. (2002), and altered to emphasize the possible role of DCN units in CMR. PC, Principal cell; TVC, tuberculoventral cell; WBI, wideband inhibitor. The PSTHs and tuning curves show a pause-build unit with sideband inhibition (top left), a narrowband auditory nerve fiber (bottom left), and a wideband onset chopper unit (right). Filled circles denote inhibitory synapses; open circles denote excitatory synapses. L, Level; $F$, frequency.

taves, with $42 \%$ of these experiments having an upper gap $\leq 0.6$ octaves. Thus, many of the experiments in this study have been done with conditions similar to the ones used in psychophysical experiments.

\section{A hypothesized neural circuit to explain CMR}

A population analysis of single units in the VCN showed that transient choppers were the only unit type that consistently showed a physiological CMR, whereas onset units primarily failed to show physiological CMR (Pressnitzer et al., 2001). A neural circuit suggested in the same study, and shown in a modified form in Figure 7, was demonstrated to be a realistic explanation of the results of Pressnitzer et al. (2001). The circuit consists of a narrowband unit, hypothesized to correspond physiologically to transient chopper units, and a wideband inhibitor corresponding to onset chopper units.

The circuit has been extended recently by Meddis et al. (2002) (Fig. 7, dotted lines), who added a narrowband inhibitory neuron corresponding to the vertical (tuberculoventral) cells found in the deep layer of the DCN, which plays the role of a delayed inhibitor of the narrowband unit. The resulting neural circuit is very similar to the neural circuit assumed to shape the responses of the principal cells in the DCN (Nelken and Young, 1994; Young and Davis, 2002). Vertical cells have been shown to correspond physiologically to the type II response pattern (Young, 1980; Voigt and Young, 1990; Rhode, 1999). For all type II units, signal representation was greatly improved in the CM condition. This would be expected from the properties of the type II cell but is at odds with a simple interpretation of the neural circuit, because the type II unit would then inhibit the response to the signal of the principal cells. Consistent with this prediction, type IV-t and type IV units were less likely to show an enhanced signal representation in the CM condition than type II units, and if the signal representation was improved, the effect was not as large as observed for type II units (compare Figs. 3, 5, and 6). Furthermore, although the response to the masker-only condition was greatly suppressed in the CM condition for many type IV or IV-t units, these units did not respond strongly to the signal. 
Principal cells in the DCN can also express a type III response pattern, and the inhibition observed in this study, in general, was not as extensive as what has been reported in the cat, with the majority of units expressing type III or type IV-t rather than type IV response patterns. The relative paucity of type IV units is a common finding in rodents and seems to be a genuine species difference (Davis et al., 1996; Stabler et al., 1996; Gdowski and Voigt, 1997; May, 2003). Type III units are better suited than type IV units to convey the signal information in a CMR-like paradigm, because they do not have a pronounced central inhibitory area (Shofner and Young, 1985; Spirou and Young, 1991; Nelken and Young, 1994; Young and Davis, 2002) and are therefore unlikely to get strong inhibitory input from type II cells. This is consistent with the majority of type III cells showing the strongest enhancement of the signal in the CM condition. Type III units in the rodent DCN could therefore be the equivalent of the narrowband cell in the VCN circuit (Pressnitzer et al., 2001; Meddis et al., 2002; Verhey et al., 2003). However, type IV units might still play a role in CMR. These units have a low-level excitatory area that is not inhibited by type II units; thus type IV units may give an enhanced response to the signal in the CM condition at low sound levels. Type IV units in the cat shape the response of the majority of type $\mathrm{O}$ units in the inferior colliculus (Ramachandran et al., 1999; Davis, 2002). However, in the guinea pig, type O response maps are less common (LeBeau et al., 2001), in agreement with the hypothesis that primarily type III units in the guinea pig DCN convey the signal information in a CMR-like paradigm.

\section{Influence of anesthetics}

Barbiturate anesthesia has been shown to strongly affect inhibition in the DCN (Evans and Nelson, 1973; Young and Brownell, 1976; Gdowski and Voigt, 1997). This is a potential confounding factor, because exploring the role of inhibition in CMR was the main objective of our study. However, using urethane (a nonbarbiturate) anesthesia, strong signs of inhibition in the DCN have been found, including several units with purely inhibitory response areas. Furthermore, 59\% of our units (entire sample, 314 units) showed spontaneous activity $>2.5 \mathrm{spikes} / \mathrm{sec}$. In the gerbil, under barbiturate anesthesia, only $12.5 \%$ of the units showed spontaneous activity $>2.5$ spikes/sec (Gdowski and Voigt, 1997).

\section{Comparison with previous physiological studies}

The responses of single units in the DCN are in close agreement with those found by Pressnitzer et al. (2001) in the VCN of the same species. The main difference between the VCN and DCN was the amount of suppression of the OFM caused by the addition of FBs. This is predictable based on the difference in strength of wideband inhibition in the different areas of the cochlear nucleus (Rhode and Greenberg, 1994). Pause-build units show the strongest inhibition, followed by chopper units, with primarylike units showing the weakest amount of inhibition.

Comparison with other physiological studies examining CMR is confounded by the use of different stimulus paradigms, different levels of the auditory pathway, and different species. Ignoring obvious species differences, in the primary auditory cortex of the cat, Nelken et al. (1999) showed that the responses of single units were strongly modulated by the masker envelope and that these modulated responses were reduced or abolished after addition of the signal. In this case, the reduction in modulation was assumed to be the cue for detection of the signal. This is in stark contrast to the responses of single units in the cochlear nucleus, in which a clear response to the signal is frequently observed in the presence of comodulated maskers. It is possible that the representation of the signal in the brainstem is recoded at higher levels of the auditory pathway. Such a recoding would reconcile the differences between the results in the cochlear nucleus (Pressnitzer et al., 2001; this study) and those of Nelken et al. (1999).

The role of wideband inhibition has also been investigated by Klump and colleagues in the auditory forebrain of the European starling. Flanking bands were assumed to activate inhibitory sidebands of single units or multiunits if they were $20 \mathrm{~dB}$ above the level of the OFM. In this condition, Nieder and Klump (2001) failed to observe CMR in the population response; however, a subpopulation of single units or multiunits was able to show CMR. In a similar study, Hofer and Klump (2003) used the same stimulus level for the OFM and FB. A minority of units showed CMR of similar magnitude to the CMR measured behaviorally in the same species (Langemann and Klump, 2001), but a population analysis failed to reveal significant CMR. It is possible that higher FB levels may have increased the magnitude of the CMR. Psychophysical studies on CMR do not agree on the effect of FB level relative to the OFM. Some studies show that the biggest release from masking occurs when the FBs have the same level as the OFM (Hall, 1986; McFadden, 1986; Moore and Shailer, 1991), whereas others found an increased CMR when the FBs were at higher levels than the OFM (Schooneveldt and Moore, 1987; Cohen, 1991).

Finally, a major issue is what constitutes the correct population of units. At the level of the cochlear nucleus, it is now reasonably well established that different unit types are performing different signal-processing tasks. If the VCN circuit suggested by Pressnitzer et al. (2001) and modified here is correct, it makes no sense to average the responses of all unit types. At present there is no evidence or theory to suggest how the responses of single units at higher levels of the auditory pathway should be combined, but if perception is governed by the response of the most sensitive units, then all physiological studies have found a CMR. The mechanism, however, remains unresolved.

\section{References}

Cohen MF (1991) Comodulation masking release over a three octave range. J Acoust Soc Am 90:1381-1384.

Davis KA (2002) Evidence of functionally segregated pathway from dorsal cochlear nucleus to inferior colliculus. J Neurophysiol 87:1824-1835.

Davis KA, Ding J, Benson TE, Voigt HF (1996) Response properties of units in the dorsal cochlear nucleus of unanesthetized decerebrate gerbil. J Neurophysiol 75:1411-1431.

Delahaye R (1999) Across-channel effects on masked signal thresholds. PhD thesis, University of Essex.

Eddins DA, Wright BA (1994) Comodulation masking release for single and multiple rates of envelope fluctuation. J Acoust Soc Am 96:3432-3442.

Evans EF, Nelson PG (1973) The responses of single neurones in the cochlear nucleus of the cat as a function of their location and the anaesthetic state. Exp Brain Res 17:402-427.

Gdowski GT, Voigt HF (1997) Response map properties of units in the dorsal cochlear nucleus of barbiturate-anesthetized gerbil (Meriones unguiculatus). Hear Res 105:85-104.

Godfrey DA, Kiang NY, Norris BE (1975) Single unit activity in the dorsal cochlear nucleus of the cat. J Comp Neurol 162:269-284.

Grose JH, Hall JW (1989) Comodulation masking release using SAM tonal complex maskers-effects of modulation depth and signal position. J Acoust Soc Am 85:1276-1284.

Hall JW (1986) The effect of across-frequency differences in masking level on spectrotemporal pattern-analysis. J Acoust Soc Am 79:781-787.

Hall JW, Haggard MP, Fernandes MA (1984) Detection in noise by spectrotemporal pattern-analysis. J Acoust Soc Am 76:50-56.

Hicks ML, Bacon SP (1995) Some factors influencing comodulation masking release and across-channel masking. J Acoust Soc Am 98:2504-2514. Hofer SB, Klump GM (2003) With- and across-channel processing in audi- 
tory masking: a physiological study in the songbird forebrain. J Neurosci 23:5732-5739.

Langemann U, Klump GM (2001) Signal detection in amplitude modulated maskers. I. Behavioural auditory thresholds in a songbird. Eur J Neurosci 13:1025-1032.

LeBeau FEN, Malmierca MS, Rees A (2001) Iontophoresis in vivo demonstrates a key role for $\mathrm{GABA}_{\mathrm{A}}$ and glycinergic inhibition in shaping frequency response areas in the inferior colliculus of guinea pig. J Neurosci 21:7303-7312.

Lloyd MEA (2002) A fast inexpensive stimulus presentation and data acquisition system for auditory neuroscience. Int J Audiol 41:263.

May BJ (2003) Species differences in the spectral processing pathways of the inferior colliculus. Paper presented at 26th Midwinter Research Meeting of the Association for Research in Otolaryngology, Daytona Beach, FL, February.

McFadden D (1986) Comodulation masking release-effects of varying the level, duration, and time-delay of the cue band. J Acoust Soc Am 80:1658 1667.

Meddis R, Delahaye R, O'Mard L, Sumner C, Fantini DA, Winter I, Pressnitzer D (2002) A model of signal processing in the cochlear nucleus: comodulation masking release. Acta Acustica United Ac 88:387-398.

Merrill E, Ainsworth A (1972) Glass coated platinum tipped tungsten microelectrodes. Med Biol Eng 10:662-672.

Moore BCJ, Shailer MJ (1991) Comodulation masking release as a function of level. J Acoust Soc Am 90:829-835.

Moore BCJ, Glasberg BR, Schooneveldt GP (1990) Across-channel masking and comodulation masking release. J Acoust Soc Am 87:1683-1694.

Nelken I, Young ED (1994) Two separate inhibitory mechanisms shape the responses of dorsal cochlear nucleus type IV units to narrow-band and wide-band stimuli. J Neurophysiol 71:2446-2462.

Nelken I, Rotman Y, Bar Yosef O (1999) Responses of auditory-cortex neurons to structural features of natural sounds. Nature 397:154-157.

Nieder A, Klump GM (2001) Signal detection in amplitude-modulated maskers. II. Processing in the songbird's auditory forebrain. Eur J Neurosci 13:1033-1044.

Pressnitzer D, Meddis R, Delahaye R, Winter IM (2001) Physiological correlates of comodulation masking release in the mammalian ventral $\mathrm{co}-$ chlear nucleus. J Neurosci 21:6377-6386.

Ramachandran R, Davis KA, May BJ (1999) Single-unit responses in the inferior colliculus of decerebrate cats. I. Classification based on response maps. J Neurophysiol 82:152-163.

Rhode WS (1999) Vertical cell responses to sound in cat dorsal cochlear nucleus. J Neurophysiol 82:1019-1032.
Rhode WS, Greenberg S (1994) Lateral suppression and inhibition in the cochlear nucleus of the cat. J Neurophysiol 71:493-514.

Rhode WS, Kettner RE (1987) Physiological study of neurons in the dorsal and posteroventral cochlear nucleus of the unanesthetized cat. J Neurophysiol 57:414-442.

Rhode WS, Smith PH (1986) Encoding timing and intensity in the ventral cochlear nucleus of the cat. J Neurophysiol 56:261-286.

Rhode WS, Smith PH, Oertel D (1983) Physiological-response properties of cells labeled intracellularly with horseradish-peroxidase in cat dorsal cochlear nucleus. J Comp Neurol 213:426-447.

Schooneveldt GP, Moore BC (1987) Comodulation masking release (CMR): effects of signal frequency, flanking-band frequency, masker bandwidth, flanking band level, and monotic versus dichotic presentation of the flanking band. J Acoust Soc Am 82:1944-1956.

Schooneveldt GP, Moore BC (1989) Comodulation masking release for various monaural and binaural combinations of the signal, on-frequency, and flanking bands. J Acoust Soc Am 85:262-272.

Shofner WP, Young ED (1985) Excitatory/inhibitory response types in the cochlear nucleus: relationships to discharge patterns and responses to electrical stimulation of the auditory nerve. J Neurophysiol 54:917-939.

Spirou GA, Young ED (1991) Organization of dorsal cochlear nucleus type-iv unit response maps and their relationship to activation by bandlimited noise. J Neurophysiol 66:1750-1768.

Stabler SE, Palmer AR, Winter IM (1996) Temporal and mean rate discharge patterns of single units in the dorsal cochlear nucleus of the anesthetized guinea pig. J Neurophysiol 76:1667-1688.

Verhey JL, Pressnitzer D, Winter IM (2003) The psychophysics and physiology of comodulation masking release. Exp Brain Res 153:405-417.

Voigt HF, Young ED (1990) Cross-correlation analysis of inhibitory interactions in dorsal cochlear nucleus. J Neurophysiol 64:1590-1610.

Winter IM, Palmer AR (1995) Level dependence of cochlear nucleus onset unit responses and facilitation by second tones or broad-band noise. J Neurophysiol 73:141-159.

Young ED (1980) Identification of response properties of ascending axons from dorsal cochlear nucleus. Brain Res 200:23-37.

Young ED, Brownell WE (1976) Responses to tones and noise of single cells in dorsal cochlear nucleus of unanesthetized cats. J Neurophysiol 39:282-300.

Young ED, Davis KA (2002) Circuitry and function of the dorsal cochlear nucleus. In: Integrative functions in the mammalian auditory pathway (Oertel D, Fay RR, Popper AN, eds), pp 160-206. New York: Springer.

Young ED, Robert JM, Shofner WP (1988) Regularity and latency of units in ventral cochlear nucleus-implications for unit classification and generation of response properties. J Neurophysiol 60:1-29. 